\title{
Medio siglo de desarrollo en la Amazonia: jexisten esperanzas para su desarrollo sustentable?
}

\author{
MARC J. DOUROJEANNI
}

$\mathrm{N}$

ESTE TRABAJO se pasa revista a los cambios ocurridos en los últimos 50 años de historia amazónica, analizando lo que tienen de positivo y de negativo en sus aspectos sociales, económicos y ecológicos, en particular extrayendo las lecciones que permitan acceder al paradigma de la sustentabilidad a través de la conjugación de la globalización y de la afirmación local o, en términos más generales, rescatando los elementos que puedan contribuír a la definición de un nuevo proyecto amazónico y a la identificación de las estrategias que podrían conducir a su implantación.

En el trabajo se parte del principio que no existe dicotomía ni conflicto real entre desarrollar y proteger y que existen posibilidades concretas de alcanzar bienestar durable en la Amazonia combinando justicia social con prudencia ecológica y eficiencia económica. Los problemas concretos decurrentes de la aplicación de esos principios son también analizados.

\section{Cambios conceptuales y nuevas percepciones}

sobre la Amazonia y el desarrollo amazonico

Enfoques nacionales: de la "conquista, ocupación y explotación» al «desarrollo sustentable»

Ha habido un cambio considerable en los enfoques teóricos sobre desarrollo amazónico. En el último medio siglo se ha pasado del concepto de conquista, ocupación y explotación, tan frecuente hasta los años 50, al de desarrollo sustentable actual, pasando por el desarrollo y el desarrollo racional de los años 60 y el ecodesarrollo de los años 70 y 80.

La visión de la Amazonía como territorio a ser conquistado, ocupado y explotado estaba íntimamente ligada a las teorías geopolíticas originadas 
esencialmente en círculos militares. Este enfoque, que dominó la primera mitad del siglo XX, tuvo expresiones diversas que incluyeron hasta conflictos armados y, en sus versiones más convencionales, grandes programas de colonización dirigidos por el Estado a lo largo de carreteras cuyos trazados respondían a criterios de ocupación territorial. Brasil y Perú fueron los paises que más usaron estas estrategias, con experiencias como las implantadas en la Trans-Amazónica y la BR-364 (Rondonia) en Brasil y a lo largo de la carretera Marginal de la Selva en Perú. Poco a poco, la visión geopolítica del desarrollo amazónico fue impregnada de criterios de integración económica que, hoy en día, son predominantes, aunque la primera nunca fue realmente abandonada.

Cuando, a finales de los años 60 y durante las dos décadas siguientes se habló de desarrollo racionaly de ecodesarrolloy, finalmente, en esta década, de desarrollo sustentable, armonizando criterios económicos, sociales y ecológicos, tanto los intelectuales cuanto los políticos rápidamente absorbieron el mensaje y pasaron a usarlo intensamente, tiendo hasta sido incorporado a textos legales y a denominaciones burocráticas, existiendo inclusive ministerios y secretarias de desarrollo sustentable.

\section{La intelectualidad \\ amazónica adbiere al desarrollo sustentable}

En la propria Amazonia se dieron los mismos cambios que a nivel nacional. Especialmente los niveles universitarios y científicos, así como los tratados y acuerdos internacionales (por ejemplo, el Tratado de Cooperación Amazónica) adoptaron el paradigma del desarrollo sustentable y realizaron experiencias muy positivas pretendiendo demostrar su viabilidad. Simultáneamente, la mayor parte de las autoridades políticas locales abrazaron el lenguage de la sustentabilidad aunque continuaron practicando desarrollo convencional, sin miramientos por los impactos sociales y ambientales.

\section{Mayor preocupación a nivel internacional}

A nivel internacional, pero también a nivel de los proprios paises amazónicos, otro cambio importante ha sido la realización de que, a pesar de su enormidad, la Amazonia está en peligro real de desaparecer, tal como se la conoce hoy, en un plazo relativamente corto. Esa información ha sido aportada por científicos y divulgada globalmente, frecuentemente distorsionada y exagerada, creando una onda mundial de preocupaciones derivadas del rol que tiene la Amazonia en los grandes ciclos bio-geo- 
químicos, especialmente en cuanto a fijación de carbono y como fuente de recursos de biodiversidad. El llamado paradigma indígena, es decir los nativos como detentores de un valioso saber ancestral y protectores del bosque, se sumó a las inquietudes del público. La reacción del público y de los políticos de Europa y Norte América, y por ende de las agencias internacionales, ha sido aumentar la presión sobre los gobiernos de los paises amazónicos para que el desarrollo sea más sustentable y aumentar ligeramente la prioridad de la Amazonia en la asignación de recursos, frecuentemente a través de una míriada de organizaciones no gubernamentales.

\section{Mitos que se derrumbaron durante las últimas décadas}

La investigación científica también demostró que muchas de las afirmaciones que se hacían sobre la Amazonia eran apenas mitos o historias muy mal contadas. Caieron sucesivamente los mitos: Amazonia pulmón del mundo, Amazonia vacía, Amazonia virgen, Amazonia homogénea y, Amazonia de tierras estériles (IDB/UNDP/TCA, 1992). Por ejemplo, la caída del mito de la virginidad de la Amazonia fue un duro golpe para los naturalistas románticos. En efecto, hoy se sabe que gran parte de esa región ha sido densamente poblada $\mathrm{y}$, en consecuencia, explotada desde hace milenios, habiéndose inclusive encontrado vestigios de grandes desarrollos culturales en Brasil, Perú y Bolivia (Meggers, 1971; Denevan, 1984; Roosevelt, 1987).

\section{Mitos sobrevivientes}

Pero algunos mitos sobreviven saludables y hasta crecen, como que la Amazonia puede y debe ser la solución para los problemas sociales de regiones periféricas, como los Andes, el Nordeste o el Sur del Brasil. También perdura el mito de que aún hay intenciones de internacionalizar la Amazonia para conservarla. Este mito es particularmente irritante, ya que sus expresiones emanan de fracciones minúsculas y frecuentemente ridículas de la sociedad de los paises desarrollados, a las que nadie da crédito en esos paises, pero que reciben un eco desmesurado en los segmentos patrioticos de los paises amazónicos. Pocos de los que acreditan en ese mito explican las motivaciones que puedan existir para internacionalizar la Amazonia, ni tampoco en que consistiría esa internacionalización. Olvidan que los servicios ambientales de la Amazonia, como la fijación de carbono o la conservación de la biodiversidad, no son únicos a esta parte del mundo y que, con los criterios que usan, existirían muchas otras partes del planeta a internacionalizar, por ejemplo, las inmensas reservas boscosas de Rusia. 


\section{Constataciones nuevas:}

\section{la Amazonia "urbana» y la Amazonia "minera»}

Durante mucho tiempo, especialmente en virtud del mito de la Amazonia vacía, pasó desapercibido un fenómeno que ahora es omnipresente. La Amazonia se ha urbanizado tanto que aproximadamente el $60 \%$ de la población habita en ciudades. Estas ciudades, algunas de ellas con más de un millón de habitantes, como Belém do Pará y Manaus y muchas ya muy grandes como Iquitos, Pucallpa, Santa Cruz, Leticia, Porto Velho, Rio Branco y Santarém, entre otras más, crecieron en promedio mas de 50 veces desde 1940, y continúan creciendo a tasas que superan toda posibilidad de proveerlas con los servicios necesarios. Aunque los deficit en cuestiones de educación y salud son grandes, aun mayores son las carencias de agua, desague, plantas de tratamiento, drenajes y manejo de residuos sólidos. La falta de pavimentación crea severos problemas de contaminación del aire por polvos sedimentables durante la estación seca. Gran parte de la población urbana sufre de pobreza crítica y habita en favelas, barriadas o villas miseria que, en muchos casos, son aglomerados de palafitos o casas flotantes.

Asimismo, por razones no muy claras, el público e inclusive los intelectuales del desarrollo amazónico no absorbieron la importancia minera y energética de la Amazonia y, tocando esos temas, lo hicieron siempre como algo colateral y en gran medida como perjudicial para el desarrollo. La imagen de los garimpeiros o gambusinos pululando en Mesa Pelada, desprendiendo toneladas de mercurio en Rondônia o invadiendo las tierras Yanomami en Roraima, en Brasil o sus equivalentes en el Pachitea y en Madre de Dios, en Perú, hicieron olvidar la minería industrial de Vale do Rio Doce o los enormes emprendimientos petroleros de la Amazonia del Perú y de Ecuador. Hoy ya es evidente que la Amazonia es un emporio minero y que tiene considerable importancia energética, tanto en términos de petróleo cuanto de hidroenergía. Es igualmente evidente que el futuro amazónico no puede disociarse de estas realidades.

\section{El creciente segmento urbano amazónico}

tiene objetivos no necesariamente compatibles con el desarrollo sustentable de la región

Especialmente en los paises andino-amazónicos, la Amazonia todavía es vista por la mayoría como una tierra donde se va a hacer dinero para luego vivir bien en otra parte. No se vá a la Amazonia para radicarse en ella, ni para desarrollarla, construirla, transformarla en una nueva nación. Se la 
ve como un sitio a anexar a la patria, casi del mismo modo en que los españoles veian a América en la época colonial. En el Brasil, esa visión también existe pero es menos fuerte que en los paises andinos y es practicada más por los grandes empresarios del Sur, que apenas consideran la Amazonia como fuente de materia prima o como lugar para especular con su dinero.

Pero, en el Brasil, la gente más humilde o los profesionales jóvenes ahora migran a la Amazonia para lá residir y, con el gran desarrollo urbano de la región, cada vez hay más gente que se suma a los indígenas, siringueiros, ribereños y otros pobladores tradicionales.

Es decir que cada vez es mayor el segmento de la población que se siente amazónica. Eso es un gran paso para un eventual cambio en el estilo de desarrollo preponderante.

Aunque esa mudanza de actitud sobre la Amazonia es muy positiva para implantar un desarrollo más sustentable no necesariamente alcanza su objetivo, pues gran parte de la población que llega a la región se convierte rápidamente en urbana y los electores urbanos suelen ver a la selva o a las zonas rurales a través del prisma de la ciudad y de sus propios problemas.

En consecuencia, ellos suelen reproducir el comportamiento de los que viven fuera de la Amazonia y, sin preocuparse de las consecuencias, sólo esperan que la selva que les rodea les de pescado, carne de monte, productos agropecuarios, madera, energía y otros bienes y servicios baratos o gratuitos.

También les importa, obviamente, recibir más y mejores servicios sociales y sanitarios para mejorar el entorno urbano. Manaus, la capital del enorme estado de Amazonas, es un buen ejemplo de esta situación. La ciudad decide qué partido va a gobernar y quien va a ser el gobernador. Recibe también casi todos los beneficios y a la población de Manaus, en general, le importa poco si hay deforestación innecesaria o si se explota madera $\mathrm{u}$ oro ilegalmente en las tierras indígenas.

Iquitos, la capital del también grande Departamento de Loreto, en el Perú, reproduce exactamente esa realidad. Sin embargo, la concentración de la población en las ciudades podría ser considerada, bajo cierto ángulo, como positiva para reducir la presión sobre los recursos naturales.

Es decir que a pesar de mejoras significativas en los aspectos conceptuales, también en la actitud local de la población y a pesar de muchos importantes y loables esfuerzos de los que se hablará más adelante, el desarrollo amazónico se mantiene igual que antes y tiene poco de sustentable. 


\section{Lo que cambió y no cambió \\ en los hechos, en el desarrollo amazónico}

Lamentablemente, los cambios positivos alcanzados durante el último medio siglo en materia de conocimientos, conceptos y enfoques sobre el desarrollo amazónico, tanto a nivel local y nacional cuanto internacional no se han plasmado en decisiones políticas y acciones suficientemente significativas como para alterar el curso del desarrollo de la Amazonia que continúa siendo esencialmente basado en el mismo criterio de conquista, ocupación y explotación que se aplica desde los primeros contactos europeos con esa región. En parte por eso, los impactos sociales y ambientales negativos continúan creciendo rápidamente en la región.

\section{La población creció a un ritmo acelerado y también la economía, pero no tanto la calidad de la vida}

Estimados de 1994 indican que la población amazónica era ya de más de 22 millones de personas, de las que casi un millón son indígenas y el 60\% de esa población es urbana. Manaus, fundada en 1848, tiene hoy más de 1.6 millones de habitantes y sigue creciendo. En todos los paises, aunque en unos más que en otros, la economía de la región amazónica ha crecido paralelamente a los índices demográficos. La mayor parte de ese crecimiento se ha debido a la expansión de las actividades agropecuaria y de la minería; por cierto, también a la expansión del comercio y de los servicios condicentes con el aumento de la población. Poco de este crecimiento económico parece deberse a la explotación de los recursos naturales renovables, como bosques, pesca o productos forestales no maderables, aunque en muchos paises estos rubros de producción aumentaron en términos absolutos.

Como en otras regiones de los paises en que se inserta la Amazonia, el desarrollo económico no a venido acompañado de desarrollo social, de equidad o de mejora sustantiva de la vida de las poblaciones originarias $\mathrm{o}$ migrantes. Bien al contrario, las condiciones de vida de la población rural o peri-urbana son peores que antes y peores que en otras regiones (IDB/ PNUD/TCA, 1992).

\section{La deforestación con fines agropecuarios y la degradación de los bosques continuaron aumentando rápidamente y los beneficios que generan son escasos}

Las estadísticas sobre deforestación valen muy poco. Existen sobradas evidencias de que en casi todos los casos la realidad las supera largamente, 
bien sea por la dificultad de interpretar las imágenes como por un enraizado nacionalismo mal comprendido, que esconde la verdad sobre la deforestación. Además, los sensores remotos son aún incapaces de revelar la degradación de los bosques, que es un problema tan serio como la propia deforestación. Pero aún siendo así, las mismas estadísticas oficiales revelan un acelerado proceso de deforestación en todos los países amazónicos que, en la década de 1980, alcanzó casi tres millones de hectáreas de bosques densos por año (WRI, 1987). El Brasil era, dado su gran tamaño, el mayor contribuyente a la deforestación de la Amazonia, con 1.4 millones de hectáreas por año o un $45 \%$ de la deforestación total de la Amazonia. Los años 90 comenzaron con una disminución del ritmo de deforestación, especialmente en el Brasil, debido al impacto acumulado de la recesión económica de América Latina, que puso en letargo las inversiones en infrastructuras, especialmente carreteras. Pero el tiempo perdido está siendo colmado rápidamente gracias a la recuperación económica y, por eso, la deforestación volvió a su ritmo de la década pasada (1). La FAO (1993), esencialmente basada en informes oficiales, considera que de 1981 a 1990 se había perdido sólo el $4.8 \%$ de la superficie restante, lo que con todo representa nada menos que 23 millones de hectáreas.

Pero lo importante es establecer el punto de partida de la deforestación masiva que se ubica en la década de 1940. Este tema es poco comentado, pues brinda una visión menos optimista de los hechos. Apenas en el Perú, la acumulación de la deforestación de 1940 a 1990, sumó 7.5 millones de hectáreas, es decir una reducción de casi 10\% de la superficie (Dourojeanni, 1990). A nivel de toda la Amazonia se estima que entre 80 y 100 millones de hectáreas fueron deforestadas de 1940 a 1990 (simplemente adicionando datos oficiales), esto es de $9.2 \%$ a $13.2 \%$ de su superficie (IDB/UNDP/ TCA, 1992). Evidentemente, estas cifras ya están ampliamente superadas por la deforestación ocurrida de 1991 a 1997, con lo que es de esperarse que esta ya eliminó el 15\% de los bosques que existían en 1940. Pero se recuerda que esas especulaciones están basadas en informaciones oficiales, las que son desmentidas por numerosos trabajos independientes (Leopoldo, 1989; Bryant et al., 1997) cuyos resultados superan el doble de los datos aquí citados.

La deforestación de un 15\% de los bosques amazónicos, o aún del doble si se hace caso a los datos no oficiales, no es per se alarmante. Lo que es alarmante son los lugares y la forma en que ocurre:

- en tierras sin vocación agrícola, mayormente bosques de protección en los paises andino-amazónicos, con graves implicaciones sobre el comportamiento de los ríos; 
- en enormes extensiones o frentes, que abarcan unidades ecológicas completas, sin guardar un equilibrio agro-forestal que permitiría mantener los bienes y servicios del bosque;

- en varzeas, reduciendo el potencial pesquero de los ríos y, lo que es peor aún la mayor parte de la tierra deforestada está sin uso o es dramáticamente sub-utilizada y hasta ahora esa deforestación no ha generado mejorías sustantivas en la calidad de la vida de la gente que la practica.

Sea como fuere, extensas partes de la Amazonia ya no son como eran antes y nunca volverán a serlo. Son ahora paisajes agropecuarios que no difieren demasiado de los que existen en otras partes de esos mismos paises. Esa es una realidad que debe ser aceptada y que implica, además, adoptar una estrategia agraria diferente a la que se aplica tradicionalmente en la Amazonia.

\section{Caza y pesca predatorias: mucho más y peor que antes}

La caza y la pesca son actividades tradicionales en la Amazonía que, si son practicadas con mesura, pueden contribuir para siempre a la calidad de la vida de los habitantes rurales y, en el caso de la pesca, también urbanos. La caza con fines comerciales ha desaparecido oficialmente pero, aún se practica ilegalmente en todos los países. La caza de subsistencia continúa tan intensa como siempre. Existe por cierto un riesgo de extinción de ciertas especies, pero este es incomparablemente mayor a consecuencia de la destrucción total de los ecosistemas por deforestación.

La pesca es una actividad creciente en toda la Amazonia (Goulding et al., 1995). En el Brasil la producción de pescado pasó de 419 mil TM en 1969 a 959 mil TM en 1984. Estadísticas parciales para Amazonas y Pará hasta 1988, indican que este crecimiento continúa. En Loreto y Ucayali, dos departamentos amazónicos del Perú, el desembarque de pescado pasó de 2,300 TM en 1980 a 14,200 TM en 1991. Estas estadísticas no incluyen la pesca no comercial (Barthem et al., 1995). Aunque no haya evidencias de extinción de especies de la ictiofauna, hay varias cuyas poblaciones están muy disminuídas y que han desaparecido localmente.

\section{La explotación forestal está cambiando de manos y aumenta su intensidad, pero el manejo forestal sigue siendo una utopía}

En la Amazonia la explotación forestal es esencialmente de tipo selectivo, oportunista y anárquico, habiendo resistido todos los esfuerzos 
para su ordenamiento y para aplicar prácticas de manejo forestal (Dourojeanni, 1997). El volumen producido (madera en rollo) viene aumentando rápidamente. Por ejemplo, en el Brasil pasó de 10 millones de $\mathrm{m}^{3}$ en 1975 a 54.3 millones de $\mathrm{m}^{3}$ en la zafra 1990/91 (Funatura/ITTO, 1994). Algo similar ocurre en Perú, Bolivia y Ecuador. Aunque la explotación forestal difícilmente puede causar extinción de especies ocasiona, en cambio, la rarificación de muchas de ellas y su extinción comercial.

El fenómeno reciente más importante en materia de explotación forestal en la Amazonia es la invasión de grandes inversionistas extranjeros, principalmente asiáticos, para efectuar explotación forestal masiva. El proceso de invasión comenzó en Surinam y Guayana, pero se ha expandido rápidamente al Brasil (Traumann, 1997) y a otros paises de la región, causando gran preocupación pues estas empresas no ofrecen mejores garantías de manejo sustentable que la explotación de pequeña escala individual parcticada por madereros nacionales. El caso de Surinam ha sido muy bien documentado (Sizer \& Rice, 1995) y en el Brasil ha sido objeto de numerosos artículos periodísticos.

Otro problema asociado a las grandes empresas madereras es la abertura de extensas áreas de la Amazonía y su probable invasión por campesinos sin tierra y la consecuente aceleración de la deforestación en la región.

\section{La explotación minera y petrolera}

crecen continuamente, con pocos cuidados ambientales

Cobre, oro, caolin, sal gema, manganeso, niquel, zinc, fierro, bauxita, diamantes son algunos de los muchos recursos minerales de la Amazonia que ya estan siendo explotados y cuyas enorme reservas aseguran, en muchos casos, siglos de producción. El petróleo y crecientemente el gas natural ya están siendo explotados en proporciones considerables en Ecuador, Perú y Colombia y los planes de expansión son grandes. Con pocas excepciones, a pesar de mucha retórica y de algunos esfuerzos destacados pero no perfectos - como la Companhia Vale do Rio Doce en el Brasil - la explotación de estos recursos naturales no renovables se ha hecho en detrimento de los renovables, especialmente de los recursos hídricos y de su potencial ictiológico.

\section{La infrastructura vial llega}

a los últimos reductos naturales, creando nuevas amenazas

La Amazonia contaba, hasta 1991, con más de $42 \mathrm{mil} \mathrm{km} \mathrm{de} \mathrm{carreteras}$ y $2.700 \mathrm{~km}$ de vías férreas (TCA, 1991), lo que admitidamente es poco. 
Pero docenas de proyectos de carreteras nuevas en la Amazonia, que durmieron durante la década perdida de la economía están siendo relanzados y algunos de ellos ejecutados. Apenas entre Brasil y los demás paises amazónicos se registraron en la prensa brasileña, en los últimos dos años, dos proyectos con Bolivia, cuatro con el Perú, y un proyecto con cada uno de los demás paises. En el interior de cada pais amazónico existen también numerosos proyectos de carreteras nuevas o de mejoramiento de las existentes, totalizando un costo de muchos miles de millones de dólares que, ahora, se prevén serán esencialmente del sector privado.

Las carreteras son apenas un instrumento y su necesidad es indiscutible. El problema está determinado por la forma en que se usan. La historia amazónica está pletórica de desastres ecológicos y sociales y, en muchos casos económicos, asociados a las carreteras (la Marginal de la Selva en Perú o la BR-364 en Brasil, entre docenas más). Podría decirse que las lecciones fueron aprendidas pero eso sería pecar de un optimismo sin fundamento. Es más, esos desastres ocurrieron a pesar de que los financiamientos eran públicos y sometidos al escrutinio de agencias financieras multilaterales. Es poco probable que los inversionistas privados apliquen ahora las lecciones duramente aprendidas por los gobiernos y los bancos multilaterales y que, en virtud de ello, las nuevas carreteras sean mejor utilizadas que las existentes.

\section{Las áreas protegidas y las tierras indígenas no reciben el tratamiento adecuado a pesar de su rol esencial}

Quizá por ser más fácil que en otras regiones, el crecimiento de las áreas protegidas en la Amazonia ha sido considerable. De no existir prácticamente ninguna en los años 1940 se ha pasado a tener 74 áreas protegidas de uso indirecto (parques, reservas, estaciones ecológicas) en 1990, que abarcan 32.2 millones de hectáreas o 4.5\% de la Amazonia (Rojas y Castaño, 1991). A eso hay que sumar las áreas protegidas de uso directo (bosques nacionales, áreas de protección ambiental, parques y reservas indígenas), las que tan sólo en el Brasil cubren 109 millones de hectáreas (Rylands, 1991). Pero además existen numerosas reservas y asentamientos extractivistas.

El entusiasmo que semejantes números provocan a simple vista se pierde rápidamente cuando se sabe que, con muy pocas excepciones, esas áreas protegidas están abandonadas o que aún disponiendo de algún control este es incapaz de evitar invasiones por agricultores y madereros, caza, pesca, explotación de minerales y otras actividades ilícitas. Igualmente es importante saber que la representatividad ecológica de esas áreas es inade- 
cuada y que, en consecuencia, no aseguran la sobrevivencia de la diversidad genética que contienen (Dourojeanni, 1997a).

\section{Coca, cocaina y tráfico de estupefacientes}

Uno de los cambios más dramáticos ocurridos en la Amazonia en los últimos 20 años es el de la explosiva expansión del cultivo de la coca y, más recientemente, también de otros cultivos para fabricación de estupefacientes. No cabe discutir aquí las controvertidas versiones sobre la superficie que estos cultivos ilegales abarcan en cada país. Baste decir que Perú, Bolivia y Colombia son los que albergan la mayor extensión que, en cualquier hipótesis, es muy significativa. Este negocio impacta en la Amazonía y en los países que lo albergan en las vertientes ecológica, económica y social. Los impactos ecológicos corresponden al cultivo y a la producción de la droga y, resumidamente, son deforestación tanto para el cultivo ilegal como para cultivos de subsistencia; fuerte erosión de los suelos por mal manejo de estos; contaminación de las aguas y suelos por agroquímicos para controlar plagas del cultivo; invasión de áreas protegidas y correspondiente destrucción de ecosistemas únicos y de la biodiversidad que contienen; grave contaminación de los cursos de agua por el uso de grandes volúmenes de diversas sustancias tóxicas para preparar la droga, en especial la pasta básica de cocaína (Dourojeanni, 1992). A lo anterior debe sumarse la contaminación generada por el combate al narcotráfico mediante herbicidas. El impacto en la economía se hace sentir, como bien se sabe, a niveles nacional e internacional, siendo su principal impacto local el derivado del lavado de dinero ilegal en base a industrias y comercios locales, en especial la industria forestal, causándole graves perjuicios. El impacto social es mayúsculo, desde el aumento de consumo de la droga y el correlacionado aumento de la violencia hasta el financiamiento de movimientos sediciosos y los actos de guerra entre estos y las fuerzas del orden, pasando por una generalización de la corrupción. Décadas serán necesarias para que la Amazonia se recupere del perjuicio creado por estas actividades.

\section{La sociedad civil participa mucho más que antes}

Un cambio trascendente en la conducción del desarrollo en todos los paises amazónicos es la creciente participación de la sociedad organizada en las decisiones que la afectan. Los gobiernos, cediendo a siglos de verticalismo, comienzan a aceptar, hasta a facilitar y apreciar la intervención de los grupos que son afectados directa o indirectamente por sus decisiones. Se está aceptando que la democracia representativa no es suficiente y que deben existir canales participativos complementarios. En la Amazonia el 
proceso participativo ha sido más lento y difícil que en otras partes de los paises pero, en la última década, ha recuperado tiempo perdido y en, varios paises, la sociedad es más actuante y coherente en la Amazonía que en otras regiones. Tal parece ser el caso en Brasil, Bolivia, Ecuador y Perú. En Brasil, es notable el accionar de la Coordenação das Organizações Indígenas da Amazonia (Coiab), del Conselho Nacional dos Seringueiros (CNS) y de numerosas ONGs locales que, crecientemente, se agrupan en foros estatales. A nivel local son cada vez más actuantes, en especial en Perú, Bolivia y Ecuador, las cooperativas de productores, las federaciones y los sindicatos $\mathrm{y}$ hasta las asociaciones de vecinos y de barrios, en el medio urbano.

\section{Aunque lentamente, las decisiones se municipalizan cada vez más}

Por otra parte, aunándose a la mayor transparencia y participación, va creciendo el rol de la municipalidad como unidad política básica, dando mayor oportunidad a la población, aún aquella que no está organizada, de ser informada, de influir en las decisiones que le afectan y, en especial, de monitorear el cumplimiento de las mismas o, en general, de ejercer control sobre la autoridad.

\section{La comunidad internacional \\ aporta poco, pero de un modo mas consistente}

Los gritos de alarma sobre la Amazonia que se profieren en los paises desarrollados no se expresan en aporte sustanciales. Ofrecimientos de miles de millones de dólares como los del G7 se transformaron en pocas centenas de millones dirigidas a uno sólo de los paises amazónicos y, curiosamente, no a aquel cuyos recursos de biodiversidad corren más peligro. El uso de esos recursos, además, está condicionado a reglas complejas que, sumadas a los entraves burocráticos nacionales, ha demorado sustancialmente la ejecución. Otras fuentes ofrecidas durante la Unced, en 1992, no se han materializado o, como en el caso de la Facilidad Ambiental Global (GEF), se han mantenido en niveles mínimos. Otras fuentes más tradicionales, como las cooperaciones técnicas multilaterales o bilaterales, se han reducido en virtud de los aportes al G7 y al GEF o se han apartado de la Amazonia, región que frecuentemente se considera privilegiada en relación a otros biomas mas amenazados, como los Bosques Atlánticos o el Cerrado del Brasil.

Otro aspecto, igualmente contradictorio en la actitud de los paises desarrollados es su dualidad de comportamiento que incluye críticas al estilo 
de desarrollo imperante en la Amazonía y condicionalidades diversas, al mismo tiempo que sus gobiernos estimulan inversiones en explotación forestal, minera y petrolera así como en turismo que, como se dijo antes, rara vez son consistentes con las premisas del desarrollo sustentable que ellos pregonan.

Pese a lo anterior hay que reconocer que los aportes de recursos donados a la Amazonia, al igual que los empréstimos multilaterales, son de mejor calidad que antes, en especial porque incluyen niveles de transparencia y participación sin precedentes.

\section{Los gobiernos se organizan cada pez mejor:}

el Tratado de Cooperación Amazónica

$y$ otros instrumentos regionales empiezan a dar frutos

Los difíciles comienzos del Tratado de Cooperación Amazónica (TCA) parecen superados. Años de reuniones estériles, frecuentemente siempre de las mismas personas, sobre temas a los que pocos daban importancia en los paises, terminaron y desde las estadías de la Secretaría Pro-Témpore en Quito y en Lima, comenzaron a discutirse asuntos cada vez más serios con personas mejor entendidas. La calidad de los documentos producidos aumenta día a día y ahora son útiles. Pero, para que el TCA rinda lo que se espera de el, su Secretaría debe estabilizarse, tal como felizmente ya fue decidido. Los acuerdos bilaterales para temas amazónicos concretos proliferan entre los paises y, en general, se desarrollan en buena armonía y para beneficio mutuo, aunque no siempre basados en criterios de sustentabilidad.

\section{Finalmente, en medio de muchos fracasos hay cada vez mayor número de experiencias con resultados alentadores}

Así como muchas de las iniciativas privadas o públicas en la Amazonia no tuvieron éxito, un número respetable de ellas si lo fueron y están documentadas. Sin embargo, hay que reconocer que mucho de lo documentado triunfalmente deja de existir sin pena ni gloria. Un buen ejemplo es el caso del manejo forestal en el Palcazú, Perú, presentado como modelo ejemplar y que, apenas terminado el apoyo externo, dejó de funcionar. Otro ejemplo fue el zoneamiento ecológico-económico de Rondonia desarrollado por el Polonoroeste que, como tantos otros ejercicios de zoneamiento en Rondonia y otros estados, comenzó bien pero nunca fue implantado a pesar de tener inclusive respaldo legal. Muchas instituciones locales, como la Funtac en el Acre, tuvieron un breve periodo de aportes 
notables al desarrollo sustentable y luego pasaron a ser insignificantes. Los éxitos iniciales y breves, seguidos de fracaso, son numerosísimos en cada país amazónico y, aunque las causas de los fracasos no son siempre imputables al concepto de la iniciciativa, es evidente que en cada caso se dejaron de examinar los intereses exógenos y los conflictos que formaban su entorno.

Cada país desde hace décadas prepara regularmente planes de acción, estrategias y/o políticas sectoriales o globales para el desarrollo de sus porciones amazónicas. En su mayoría, estos planes están razonablemente bien hechos y contienen, especialmente en sus versiones más recientes, elementos de sustentabilidad del desarrollo. Algunos de esos trabajos, sean esfuerzos públicos o privados, son notables como por ejemplo el análisis Politicas Públicas Coerentes para uma Amazônia Sustentável preparado por el Grupo de Trabajo Amazónico en el Brasil (GTA/Amigos da Terra, 1994). Pero también, cada vez, estos esfuerzos se quedan esencialmente en el papel y su contribución no pasa de crear, lentamente, conciencia sobre el problema.

Pero otras iniciativas tuvieron más éxito, por ejemplo la eliminación parcial, en el Brasil, de los incentivos fiscales para actividades que estimulan la deforestación en la Amazonia, como la pecuaria. Proyectos como el denominado RECA en el Brasil, Las Gaviotas en Colombia o el Programa de Primates del Perú mantienen su éxito a través del tiempo. Otras iniciativas, a veces lanzadas por el sector público, son rápidamente adoptadas para beneficio general. Entre estos éxitos merecen mención la rápida domesticación y aprovechamiento del camu camu (Myrciaria dubia), primero en el Perú y ahora en el Brasil, del cupuazú (Theobroma grandiflorum) en el Brasil, de la uña de gato (Uncaria tomentosa) en el Perú y la difusión del cultivo del pijuayo (Bactris gasipaes), entre otras plantas amazónicas de alto potencial económico.Los conceptos de agroforestería y los de cultivos consorciados (o agricultura promiscua), aunque no realmente nuevos en la Amazonia, fueron, asimismo, bien recibidos por los productores que ahora los aplican en uma escala significativa. La piscicultura también empieza a ser aceptada en la Amazonía.

\section{¿Porqué no se aplica}

lo que se sabe para desarrollar la Amazonia?

En este capítulo se pretende analizar las razones por las que tantas buenas ideas sobre el desarrollo amazónico, incluído el desarrollo sustentable, no prosperan o no prosperan más rápido. Es contradictorio, en efecto, que una estratégia de desarrollo que, en el papel, parece tan positiva a todos los actores no despierte más entusiasmo y más adhesión. 
Marcelo Carnaval - Agência O Globo - 11.8.98

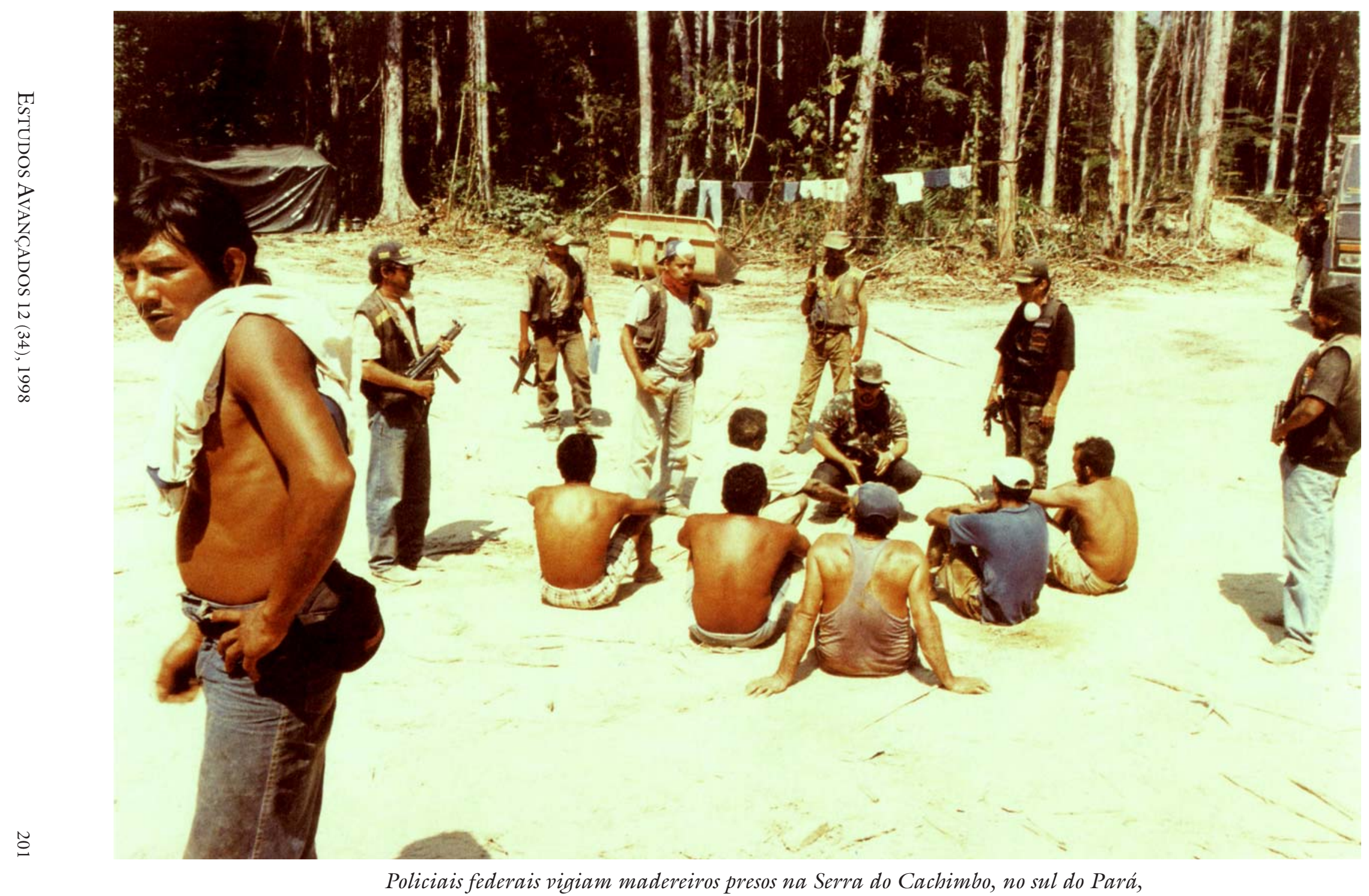
que contavam com a ajuda dos indios caiapós na extração ilegal de mogno. 


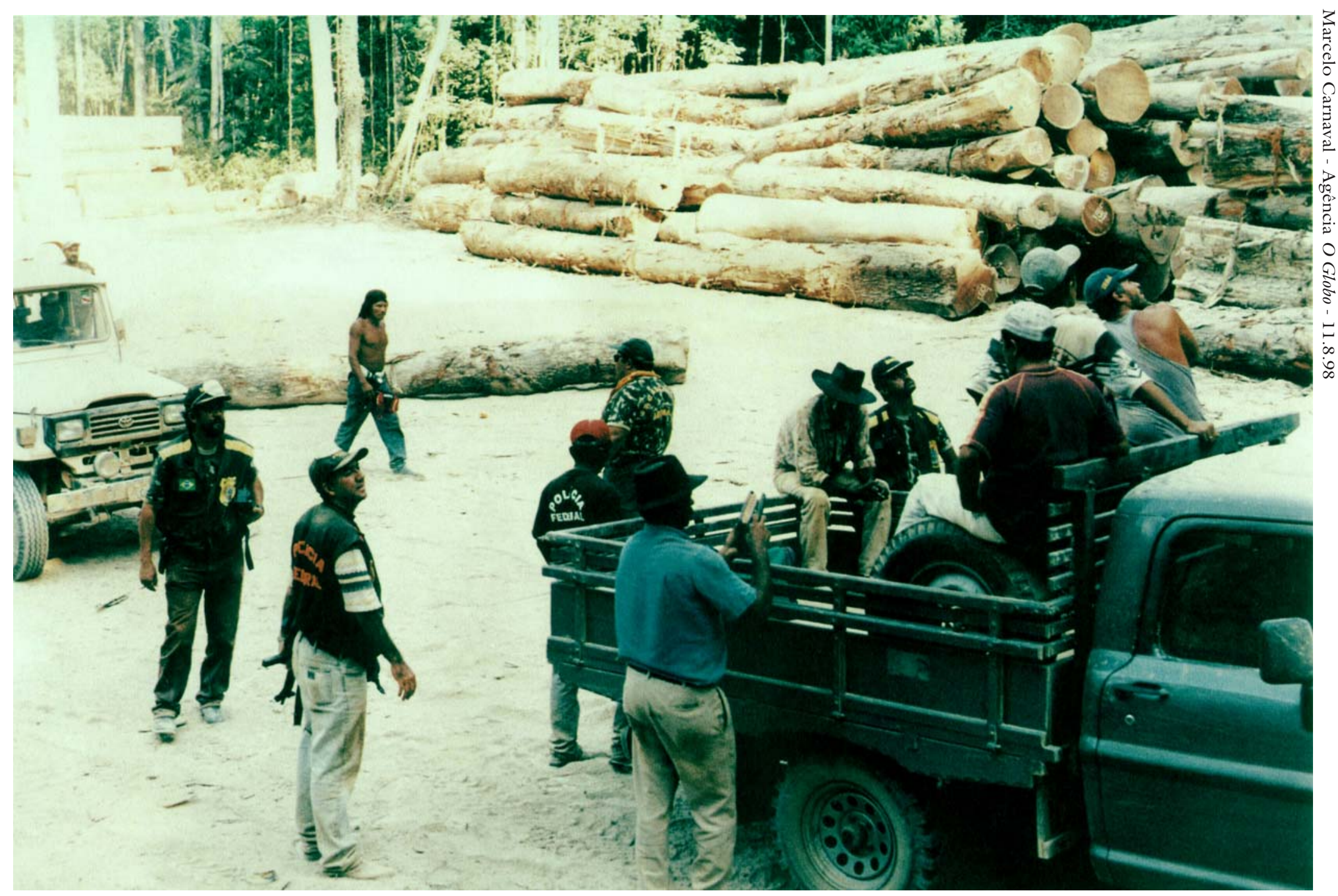

Policiais federais guardam os madereiros presos durante a operação no sul do Pará e colocados na carroceria do caminhão. Ao fundo, as toras prontas para serem retiradas da reserva dos indios caiapós. 
Entre las causas se han identificado algunas:

- la imprecisión del concepto que es controversial hasta en los mismos medios intelectuales que lo crearon;

- el desconocimiento general de la nueva propuesta, aún poco divulgada a nivel popular y la desconfianza que provocan ideas nuevas, en especial despues del escaso éxito de conceptos similares que lo antecedieron;

- los intereses conflictivos del influyente segmento social que se considera afectado negativamente por el desarrollo sustentable, entre ellos muchos agricultores, los madereros, los pescadores comerciales, los mineros informales y muchos formales y, los campesinos sin tierra;

- la incapacidad del Estado, en sus ramas ejecutiva, legislativa y judicial, para conducir el proceso de desarrollo, brindando tanto los alicientes como los correctivos;

- la dificultad, para muchos, de percibir la degradación del ambiente amazónico;

- la existencia de indicadores de éxito no siempre adecuados. Pero esa enumeración, aunque explica en parte el problema, no es completamente satisfactoria.

\section{No existe un paradigma bien definido, concensuado $y$ atractivo de desarrollo sustentable para la Amazonia}

La visión de los científicos, expertos y hasta cierto punto de los habitantes tradicionales sobre como desarrollar la Amazonia difiere radicalmente de la visión de la mayoría de los políticos, de los empresarios e inversionistas, de los habitantes urbanos y del pueblo que emigra a la región.

El segundo grupo domina política y económicamente imponiendo su visión y sus estrategias sobre los primeros. Estos, grosso modo, abrazan o tienen objetivos compatibles con la teoría del desarrollo sustentable. Los segundos, como ya se mencionó, o no la entienden o simplemente la rechazan y, peor aún, usan la retórica del desarrollo sustentable para encubrir estilos de desarrollo que no son sustentables, originando gran confusión.

Pero se cometería un error en asumir que el primer grupo es monolítico pues hay casi tantas interpretaciones de desarrollo sustentable como de personas hablando de eso. 
De lo anterior se desprende, por una parte, que pocos saben qué es desarrollo sustentable y menos aún saben como expresarlo en acciones de desarrollo que no sean las consabidas restricciones ambientales que, aunque necesarias, por no ir acompañadas de sus contrapartes de desarrollo, son mal recibidas. De otra parte, muchas iniciativas de desarrollo sustentable que combinan efectivamente producción y productividad con los procesos ecológicos enfrentan una estructura económica que les es completamente defavorable. Todo indica que el concepto de desarrollo sustentable, a pesar de los esfuerzos desarrollados, aún no tiene los pies en el suelo y no lleva suficientemente en cuenta los anhelos populares que interpretan los políticos ni los intereses económicos de los inversionistas. También es posible que el desarrollo sustentable no tenga ninguna oportunidad efectiva de prosperar dentro del marco de la economía neoliberal y dentro del marasmo social actual.

En efecto, gran parte de las dificultades del concepto de desarrollo sustentable parten de lo que algunos llaman la "trampa conceptual" de aceptar que desarrollo sustentable es inseparable de crecimiento (2). Esto es refutado por un número creciente de economistas. Sin embargo, debe reconocerse que el rechazo al desarrollo sustentable sería aún mayor, por parte del establishment economíco y político si no se recurriera a esa argucia.

\section{Los conflictos de intereses de los actores amazónicos no se estudian ni resuelven}

La identificación de los actores y de sus intereses y de la forma de conciliarlos en el marco del desarrollo sustentable parece faltar en la mayoría de los ejercicios hechos.

Los grupos de actores amazónicos o endógenos, grosso modo, son los habitantes amazónicos tradicionales (ribereños, indígenas, extractivistas); los agricultores pobres llegados recientemente de otras partes del país; los hacendados o agricultores ricos; los campesinos sin tierra; los garimpeiros; los industriales; los comerciantes y proveedores de servicios; los habitantes urbanos; un grupo que incluye las organizaciones no gubernamentales, las universidades y entidades científicas. Además, hay numerosos actores exógenos, también muy influyentes.

Apenas como ejemplo, en el grupo de los pobladores tradicionales hay intereses sumamente contradictorios, pues si bien todos ellos podrían asimilar con beneficio mucho de los parámetros del desarrollo sustentable, varios, como los madereros y pescadores, son perjudicados en el corto plazo. Para estos últimos, el manejo del bosque o de la pesca simplemente implica 
prohibiciones y restricciones, es decir ganar menos y tener más problemas. Para los agricultores, la obligación de conservar una parte de sus tierras bajo cobertura forestal es percibida como un perjuicio, no como un beneficio... y así sucesivamente.

Si la propuesta de desarrollo no lleva en cuenta todos esos intereses conflictivos y si no los armoniza, o por lo menos los concilia, termina fracasando. Esa es la historia del zoneamiento en la Amazonia. Pero no basta con conciliar intereses de actores amazónicos ya que la Amazonia no es una isla y todo lo que pasa en ella está condicionado por lo que ocurre en el resto de esos mismos países. Por ejemplo, los resquemores geopolíticos que deciden, sin consulta pero con mucha publicidad, los trazos de carreteras o de operaciones costosas como las de Calha Norte o Sivam, en el Brasil también deben ser considerados y armonizados.

Las carreteras que recelan, con toda razón ambientalistas y frecuentemente también los indígenas, son un buen ejemplo de conflicto abierto. La población amazónica, lógicamente, quiere carreteras y siempre se siente lesionada por las intervenciones que proponen demorar su construcción para garantizar la conservación del patrimonio natural o la integridad de los territorios indígenas.

Traducir el desarrollo sutentable en términos que luzcan menos conflictivos y complejos, traducirlo en propuestas de acción social y económicamente claras, dando apertura a los amplios segmentos sociales que aún se oponen a él o que lo ignoran, es una tarea gigantesca pero indispensable.

\section{La creciente incapacidad del Estado agrava todo}

Los paises amazónicos nunca fueron dotados de estados eficientes pero, por coincidencia, desde que apareció la opción del desarrollo sustentable enfrentan su peor crisis, derivada en gran medida del criterio neoliberal de que la empresa privada debe asumir muchas de sus funciones y que el estado debe reducirse al mínimo pero ser más eficaz y eficiente. Esto que en teoría es loable resultó, en la práctica, un desastre ya que en efecto el estado se ha comprimido pero ni su eficiencia ni su eficacia aumentaron debido a que los salarios de los funcionarios públicos continuan reduciéndose y a que no se les ha provisto de los medios para trabajar (Naim, 1994).

Lo grave es que mientras eso ocurre con el poder ejecutivo, las pretendidas reformas del poder judicial van por el mismo camino que las del ejecutivo y que lamentablemente el poder legislativo continúa hundido 
en política poco constructiva. De ese modo, intentar montar una política coherente para la Amazonia es misión imposible. Y peor aún, esa situación va generando una cierta anarquía social que, en la Amazonia, es palpable.

\section{A pesar que la Amazonia "natural» se contrae rápidamente, los que moran en ella no lo perciben}

Esto es un fenómeno normal pero que obstaculiza mucho obtener una actitud positiva de la población para cambiar de estilo de desarrollo. Los europeos o, por ejemplo, los habitantes de São Paulo tienen sin duda más conciencia de lo que ocurre en la Amazonia que los propios moradores de ésta, gracias a la globalización de la información, a un mayor acceso a ésta y a un nivel educativo superior.

\section{Los riesgos de privatizar recursos naturales renovables}

La reducción de la función del Estado en el manejo de la economía es una meta razonable y deseada. Sin embargo, en su aplicación inicial está acompañada de excesos tales como los que están siendo promovidos en América Latina. La nueva legislación propuesta en Perú sobre agua y bosques proporciona un buen ejemplo: Una nueva ley de agua establecerá un mercado libre para derechos de agua, independiente de los derechos de tierra. En esta forma, los campesinos tendrán que competir con otros usuarios del agua, tales como la industria y los consumidores urbanos, quienes pueden pagar mucho mas por el agua en un país en el cual la agricultura mas productiva se lleva a cabo en la zona costera desértica y en la zona seca andina. Se espera, por lo tanto, que la alza del precio del agua afecte a los campesinos de las tierras costeras y andinas marginales. Simultáneamente, una nueva ley forestal está permitiendo la privatización de los bosques públicos enteramente localizados en la cuenca amazónica en condiciones muy ventajosas. El efecto combinado de ambas leyes, favorecidas por las nuevas inversiones en carreteras, puede crear un nuevo flujo de migraciones de la Costa y los Andes hacia el Amazonas. La explotación forestal necesita de caminos y, como el manejo forestal aun no es rentable, es evidente que en el futuro próximo igual que en el pasado, los madereros revenderán o abandonarán la tierra a campesinos migratorios poco después de extraer la madera comercial, asegurando una deforestación masiva.

El ejemplo peruano no está aislado. Forma parte de un nuevo patrón económico que habla mucho sobre la mejoría de la pobreza, sobre el ambiente y aun sobre la conservación de bosques, pero que promueve la 
apertura de nuevas tierras y la transferencia de la propiedad pública a un sector privado que nunca ha mostrado en América Latina ninguna preocupación social, ni mucho menos intención de preocuparse de las externalidades ambientales.

¿Existe algo que pueda ser hecho para que ele desarrollo amazónico sea más sensato?

\section{Priorizar la intensificación del uso de la tierra agricola ya habilitada}

Nada nuevo existe bajo el sol: la recomendación más importante para orientar mejor el desarrollo amazónico es dar la máxima prioridad a usar bien la tierra que ya fue deforestada y que se encuentra dramáticamente sub-utilizada en toda la Amazonia (Dourojeanni, 1990). En esas tierras es esencial proveer las infrastructuras de trasporte y de apoyo técnico necesarias para alcanzar una productividad compatible con la capacidad de uso de los suelos, que es mayor que lo que generalmente se reconoce. La infraestructura social debe crearse en paralelo con el crecimiento de la economía de esas áreas. Intensificar la producción y productividad es mucho más importante que la expansión de la agricultura, la opción preferida de los demagogos.

\section{Manejo del bosque para bienes $y$ en especial para servicios ambientales}

Mantener un balance apropiado entre el área cubierta por actividades agropecuarias y la cubierta por actividades forestales o de conservación es esencial. Pero, como bien se sabe, no acontece en la práctica y la expansión agropecuaria a niveles local y regional avasalla los bosques. La principal razón es que las teorías sobre el valor económico del bosque no se materializan en la práctica, excepto a través de la explotación destructiva. El manejo forestal para productos maderables y no maderables no es rentable bajo las condiciones sociales y económicas actuales en América Latina. Los obstáculos tradicionales al manejo forestal impuestos por las nuevas dimensiones de sustentabilidad lo hacen aun menos rentable en términos financieros (Dourojeanni, 1997).

Por lo tanto, para hacer posible el manejo forestal sustentable es esencial que los servicios o externalidades ambientales generados por los bosques se tomen en cuenta y sean pagados por todos aquellos que se benefician de ellos. Entonces, los bosques sería manejados mas cuidadosamente para producir bienes - algunos de los cuales, como las maderas precio- 
sas, deben ser caras - y para poder mantener un alto nivel de generación de servicios los cuales serían vendidos a la sociedad por una tarifa, como ya se acepta pagar por el agua, alcantarillado, energía y recreación.

Los servicios que los bosques proporcionan son bien conocidos. Actualmente, aun cuando la cuestión del calentamiento global es materia de debate (Stone, 1992), el principal servicio que un bosque, entre otros ecosistemas (3) puede proporcionar, es servir de almacén para el carbono que de otra manera sería emitido a la atmósfera y como una trampa para el carbono emitido por las actividades humanas. El valor de este servicio puede calcularse en base al volumen de carbono capturado y fijado por unidad de superficie y al costo que tenga para la sociedad reducir las emisiones de carbono (Dower \& Zimmerman, 1992). Varios análisis, con base en datos disponibles sobre almacenaje de carbono demuestran que el valor de mercado de las tierras forestadas para la agricultura - o producción maderera es menor que su valor para la captura de carbono. Un documento reciente sobre la Amazonía ilustra muy bien esta opción (Schneider, 1993). Pero este servicio es adicional al de la producción de bienes.

Por lo tanto, la idea de un impuesto por carbono (Goldemberg, 1990), a ser pagado en todo el mundo (4) por cada usuario de energía fósil, el cual serviría para compensar a cualquier país o propietario que conserve bosques, podría ser la solución para hacer rentable el manejo de bosques naturales en los trópicos (5). Ya existen impuestos al carbono en unos cuantos países, tales como Suecia, Finlandia y los Países Bajos. Por supuesto, sería necesario un acuerdo mundial para desarrollar el concepto y una organización mundial tendría que supervisar su aplicación. De hecho, sería relativamente fácil con las técnicas existentes de sensoramiento remoto saber exactamente cuantas hectáreas de bosques son taladas o mantenidas en cada lugar. Hasta se podría evaluar la degradación forestal. Para administrar este impuesto, los países o regiones de un país recibirían un estado de cuenta anual con el balance de sus existencias forestales y de los pagos que les corresponden recibir y distribuir.

Por supuesto, la captura de carbono no es el único servicio que los bosques proporcionan. La provisión de agua (Salati, 1985) y el reciclado del agua son igualmente importantes, especialmente para la producción de energía, y el consumo industrial y urbano. El reciclado del agua no es un servicio natural al que es fácil valorar, aunque en principio no es diferente que la provisión de agua. En unas pocas áreas de América Latina (6), la tarifa de agua y energía incluye un porcentage que se reinvierte en el manejo de cuencas. Sin embargo, la situación usual es que las empresas que procesan 
y distribuyen agua perciben a ésta como una mercancía gratuita, y solo cobran al consumidor final por los costos directos de proporcionarla. $\mathrm{Ni}$ un centavo se dedica al manejo de cuencas. A pesar de eso, las tendencias legales actuales muestran que sería relativamente fácil que este servicio fuera reconocido y aceptado. Igual que con el servicio previo, el dinero recolectado se haría disponible directa o indirectamente a aquellos que verdaderamente manejen sus bosques o tierras siguiendo un plan de manejo.

El caso de la conservación de la biodiversidad en los bosques naturales es similar al caso de la reducción del efecto de invernadero. Sin embargo es mucho mas difícil hacer que la gente pague por este servicio, especialmente debido a que la Convención sobre la Biodiversidad establece reglas que son especialmente difíciles de aplicar para tener acceso a los recursos genéticos, incluyendo todas las complejidades inherentes en los derechos intelectuales y de patente. La solución para recompensar a los países por conservar los recursos de biodiversidad se concibió a través de fuentes de financiamiento nuevas y adicionales que cubran todos los costos incrementales de la implementación de la Convención. Hasta hoy, el Fondo para el Medio Ambiente Mundial (GEF) ha servido como el mecanismo financiero interino, pero tiene recursos desmedidamente inferiores a las necesidades, un proceso administrativo complejo para el financiamiento y carece de prioridades adecuadas para la conservación de la biodiversidad. Los prospectos de que este mecanismo contribuya a pagar por el servicio de conservación de la biodiversidad no son buenos. Probablemente sería mejor asignar un valor promedio - probablemente mas o menos arbitrario - a los recursos genéticos de valor actual, de valor a mediano plazo y de valor a largo plazo, correspondientes a cada uno de los principales ecosistemas naturales y hacer que todos los usuarios de los productos originados en la biodiversidad, en todo el mundo, paguen una tarifa que sería redistribuida en función de los mismos mecanismos sugeridos para la fijación de carbono (7). Los bosques proporcionan muchos otros beneficios públicos además de la captura de carbono y la conservación de la biodiversidad, algunos bastante tangibles tales como la seguridad contra aluviones y avalanchas, y otros mas difíciles de medir como el valor estético de los paisajes naturales, los refugios de vida silvestre, los recursos para la investigación científica y los valores puramente éticos, tales como el solo hecho de que existen.

Asegurar, por lo menos, un sistema representativo de áreas protegidas

La importancia de conservar un muestrario representativo de los ecosistemas amazónicos y de la biodiversidad que contienen es evidente y, 
generalmente aceptada. Pero es menos comprendido que si todos los esfuerzos futuros para realizar desarrollo sustentable fracasaran, un buen sistema de áreas protegidas podría ser la mejor y la principal garantía de no perder totalmente la esencia de los recursos genéticos de la región (Dourojeanni, 1997a). Por eso ese tema es tan crucial en esta época en que el proceso de transformación de los ecosistemas amazónicos naturales en completamente antrópicos se acelera rápida e inevitablemente.

\section{Acentuar el proceso \\ de democratización, incentivando más la autonomía local}

Lo esencial del progreso en materia de desarrollo sustentable en la Amazonia se ha debido a la profundización del proceso de democratización al que integran diversos elementos: la transparencia o acceso público a las información sobre decisiones oficiales, la participación directa o a través de organizaciones no gubernamentales, la desconcentración de funciones y, de la descentralización del poder, llevando al nivel regional o local decisiones que antes se tomaban en las capitales, muchas veces alejadas de la Amazonia. La llamada municipalización, en especial, se ha revelado una herramienta de gran utilidad. Pero el camino por recorrer en esa área es aún muy largo y complejo. Las deficiencias o debilidades institucionales, la escasez de personal calificado y la corrupción, entre otros problemas, son aún dominantes. La política en la Amazonia está dominada por el oportunismo, mucho más que en otras partes de los paises en que se localiza.

La educación deberá desempeñar un rol central en el proceso de democratización que conduzca finalmente a la aplicación del desarrollo sustentable. La necesidad de fortalecer la educación es a todo nivel, sin descuidar el universitario ni los indispensables esfuerzos requeridos en investigación científica y tecnológica in situ.

\section{Acabar con el criterio \\ de que la naturaleza es tierra improductiva}

Los argumentos del economicismo de corto plazo imperante en el mundo ha contaminado, en la Amazonia, a aquellos que procuran la justicia social para los que no tienen tierras. Los bosques, la naturaleza en general, es vista como tierra improductiva o desperdiciada que debe ser utilizada casi exclusivamente a través de sus sustitución por tierra productiva, es decir tierra sin árboles pero con pastos o agricultura (GTA/Amigos da Terra, 1994). Curiosamente, pocos llaman tierra improductiva a las decenas de millones de hectáreas abandonadas por la agricultura después de un efímero 
pasaje, cubiertas de purmas o capoeiras es decir de vegetación secundaria de escaso valor económico actual.

\section{Redefinir "éxito» en materia de desarrollo amazónico}

Éxito, en desarrollo amazónico, no puede ser muy diferente de éxito en cualquier otra región. Es injusto, en una región cubierta de bosques de la que se abre una porción a la agricultura, asumir que la deforestación es automáticamente un indicador de fracaso. Todo depende, como se explicó antes, de donde se deforesta y de qué ocurre con la tierra deforestada. Si la agricultura se instala donde los suelos son aproriados, si se respeta un equilibrio determinado entre áreas boscosas y áreas agrícolas, si la producción es alta o razonable, si la productividad se mantiene, si los agricultores tienen ingresos y una calidad de vida comparable a otras regiones o mejor que la que tenían antes de venir, entonces se está en presencia de un caso de éxito. Bajo esas premisas es posible deforestar un porcentaje muy significativo de la Amazonia sin amenazar los servicios ambientales. Y, en la práctica, existen muchos casos de esos en la Amazonia. Pero, si como es frecuente, las evaluaciones se centran en la tasa de deforestación, se crea una figura incongruente. Pero éxito en la Amazonia también debe, como en otros lugares, medirse en términos de crecimiento económico y de progreso social, asimismo comparando las condiciones socio-económicas de la población migrante antes y después de instalarse en la región.

\section{Reforestación y forestación para producción de madera, leña, pulpa y/o fijación de carbono}

Existe la posibilidad de que las plantaciones forestales reduzcan la presión sobre los bosques naturales siempre y cuando éstas no sean establecidas a través de la deforestación, la que es innecesaria considerando la inmensa superficie de tierra deforestada y sub-utilizada o en descanso. Estas plantaciones pueden proporcionar madera, leña y materia prima baratas para pulpa y papel permitiendo un uso mas valioso de la madera de los bosques naturales. En el Brasil existe una ley que requiere que las haciendas mantengan un procentaje de sus tierras como bosques naturales, especialmente en las orillas de ríos, laderas y cualquier otra área no apta para la agricultura. Esta legislación a pesar de que es ignorada ampliamente, está siendo aplicada por algunas grandes empresas de plantaciones forestales para conservar los remanentes de los bosques originales.

Las plantaciones para fijación y almacenamiento de carbono han sido probadas en Guatemala y Costa Rica, como parte de un concepto de imple- 
mentación conjunta desarrollado en la convención sobre Cambio Climático. La idea es que los contaminadores en los países desarrollados pueden encontrar que es más barato compensar sus emisiones de $\mathrm{CO}_{2}$ plantando o preservando árboles en otra parte y especialmente en los países tropicales. Guatemala fue uno de los primeros países que usó esta opción. En Costa Rica el proyecto Carfix ya consiguió 12 mil hectáreas que pertenecen a 95 pequeños productores en donde se llevan a cabo nuevas plantaciones, manejo forestal y protección del bosque por una ONG local en sociedad con la Wachovia Timberlad Investment Management de los Estados Unidos. Bajo este concepto, un grupo de intelectuales brasileños ha estado promoviendo un programa masivo de plantaciones forestales para capturar y fijar $\mathrm{CO}_{2}$ en Brasil. Por supuesto, las plantaciones no son siempre necesarias ya que la regeneración de bosques naturales, el manejo forestal y la protección de los bosques son igualmente importantes para lograr esta meta (Dourojeanni, 1997).

\section{Agrosilvicultura, rebabilitación de bosques degradados y manejo forestal del crecimiento secundario}

No hay mucho que añadir a estas opciones bien conocidas para conservar los recursos forestales. La promiscuidad de plantas en los sistemas agroforestales podría ser un socio importante de la conservación de la biodiversidad en los ecosistemas naturales al igual que una reserva excelente de carbono y debe ser alentada. Lo mismo es cierto para la rehabilitación de ecosistemas la cual, en términos de servicios ambientales, es excelente aún más debido a que las inversiones en rehabilitación son generalmente muy pequeñas comparadas con la reforestación. El caso mejor conocido está siendo desarrollado con éxito en la región de Guanacaste en Costa Rica. Los bosques en barbecho pueden también ser manejados para producir bienes que no tienen efectos negativos importantes sobre los servicios ambientales que proporcionan, como está siendo demostrado en Pucallpa, Perú, por los trabajos conjuntos de la Universidad de Toronto y la Universidad Nacional Agraria de La Molina, en Perú (Dourojeanni, 1987; Blain, 1996).

\section{Oportunidades para ecoturismo}

El ecoturismo, ya equivalente al 6\% de las exportaciones mundiales está creciendo. La Sociedad de Ecoturismo y otros grupos de ecoturismo indican que en 1991 el turismo ecológico generó alrededor de US\$12 billones. En la Amazonia ya existe un desarrollo significativo del ecoturismo en algunas regiones del Perú y del Brasil. En principio el ecoturismo podría 
pagar por la conservación de los bosques, sea directamente con inversiones en áreas protegidas, o indirectamente si las autoridades nacionales pudieran cobrar impuestos a esta industria difícil de controlar. Actualmente, al igual que para el turismo en general, la mayor parte de las operaciones de ecoturismo están en manos de empresas extranjeras.

\section{Conclusión}

La conclusión a sacar del examen que antecede es la misma que en análisis hechos durante los últimos 50 años: se asume saber qué hacer en el propósito de reorientar el desarrollo amazónico y hacerlo crecientemente sustentable, con todo lo que ello implica en cuanto a crecimiento económico, justicia social y prudencia ecológica, pero continúa sin saberse bien cómo hacerlo. En efecto los obstáculos, aunque con algunos cambios positivos, continúan omnipresentes. Los cambios favorables, si bien importantes, no permiten mucho optimismo considerando la re-aceleración de los procesos clásicos de explotación amazónica y algunos problemas nuevos como son el enorme peso político de la población urbana.

El cambio difícilmente podrá salir de abajo pues los habitantes rurales tradicionales de la Amazonía son proporcionalmente cada vez menos significativos y, además, porque sus anhelos inmediatos coinciden con las de los políticos tradicionales. Por ejemplo, ellos siempre y lógicamente proponen que la inversión prioritaria sean las carreteras que les comunican con el resto del mundo. El cambio, al menos el cambio pacífico, será gradual y posiblemente deberá salir esencialmente de arriba, es decir de políticos e intelectuales, como en el pasado. Las carreteras, en ese caso, siempre serían prioritarias pero en términos de su mejoramiento en áreas donde ya existen tierras abiertas a la colonización y donde su calidad sustenta la inversión. Pero para que eso acontezca se enfrenta el hecho de que la Amazonía de cada país es, asimismo, la porción menos influyente en la política y en los gobiernos. Los intelectuales escapan a la generalización anterior y en gran parte está en sus manos crear las condiciones políticas para que la Amazonia tenga la prioridad necesaria para salir del marasmo actual.

Las estrategias de acción deben evidentemente incluir un esfuerzo concentrado de educación que, aunque lenta en sus resultados, es la mejor garantía de cambio. Adicionalmente, debe aumentarse el esfuerzo de concientización de la opinión pública, especialmente la urbana en la propia Amazonia, dado su gran influencia en la política regional.

También, si realmente se consigue levantar la producción y la productividad agropecuaria, así como la minera y energética sin desmedro 
del entorno natural, lo que es posible y, si concomitantemente se consigue recaudar sobre esa base los recursos para impulsar el desarrollo sustentable, la Amazonia puede despertar por sí sóla, sin más apoyo

Notas

1 El 25 de julio de 1996 el gobierno del Brasil divulgó la información de que la deforestación creció $34 \%$ con relación a 1992, habiendo pasado de 1.1 millones de hectáreas a prácticamente 1.5 millones de hectáreas por año. Simultáneamente el gobierno dió a conocer un paquete de medidas de protección.

2 Ver el trabajo de R.P. Guimarães, presentado en la misma Conferencia.

3 Los bosques, por supuesto, no son los únicos sistemas que secuestran carbono. Un estudio reciente de los pastos en las sabanas de Sudamérica indica que los pastos con raíces profundas pueden remover hasta dos millones de toneladas de dióxido de carbono de la atmósfera cada año. Comunicado de prensa del CIAT em septiembre 1994.

4 Tal impuesto sería pagado por los ciudadanos de los países desarrollados y en desarrollo y sería distribuido para complementar los ingresos de todos aquéllos que manejan o conservan áreas forestadas, en las regiones tropicales, templadas o boreales. Este impuesto podría ser recolectado y redistribuido en muchas formas a seren discutidas por los gobiernos. Además, esta podría ser la principal y única justificación de una nueva organización forestal mundial.

5 Goldemberg (1990) calculó que un impuesto de solo US\$ 1 por cada barril de petróleo o de US\$ 6 por tonelada de carbón equivalente, generaría US\$ 50 billones al año.

6 Uno de los casos mejor conocidos es la tarifa que pagan los productores de café en Colombia para mantener un fondo para la conservación de cuencas

7 Estudio preparado por la Rural Advancement Foundation International con base en Ottawa (Conserving indigenous knowledge: integrating two systems for innovation, 1994) calculó la bio-piratería a países del Tercer Mundo en US\$ 5.400 millones por año. El GEF proporciona sólamente unos pocos cientos de millones por año a los paises en vías de desarrollo para conservar su biodiversidad.

\section{Referências bibliográficas}

ANÓNIMO. Cuando un secuestro es buena noticia. Reforma, v. 2, n. 4, jun. 1995. 
BARTHEM, R.B.; GUERRA, H. \& VALDERRAMA, K. Diagnóstico de los recursos hidrobiológicos de la Amazonia, $2^{2}$ ed. Lima, Tratado de Cooperación Amazónica (TCA), 1995. 162p.

BLAIN, D. Fertility factors limiting the growth of pioneer trees on upland fallow soils of the Amazon basin, Iquitos, Peru. Toronto, 1996. Tésis (doctoral). Departamento Graduado de la Facultad de Silvicultura, Universidad de Toronto. $142 p$.

BRYANT, D.; NIELSEN, D. \& TANGLEY, L. The last frontier forests Washington, DC, World Resources Institute, 1997. 42p.

DENEVAN, W.M. Ecological heteregoneity and horizontal zonation of agriculture in the Amazon floodplain In: M. Schmink \& Ch.H. Woods (eds.). Frontier expansion. Florida, University of Florida Press, 1984, p.311-336.

DOUROJEANNI, M.J. Una nueva estrategia para el desarrollo de la Amazonia Peruana Lima, Revista Forestal del Perú, v. 6, n. 1-2, p. 41-58, 1976.

Amazonia peruana: desarrollo y conservación Lima, La Revista, v. 1, n. 3, p. $45-49,1980$

Aprovechamiento del barbecho forestal en areas de agricultura migratoria en la Amazonia peruana Lima, Revista Forestal del Peru, v. 14, n. 2, p. 15-61, 1987.

de la Amazonia, 1990. 444p.

. The future of the Latin American Forests. Washington, DC, Inter-American Development Bank, Environment Division, 1997. 25p.

Areas protegidas: problemas antignos y nuevos rumbos Washington, DC, Banco Interamericano de Desarrollo, 1997a. 31p.

DOWER, R.C. \& ZIMMERMAN. M.B. The right climate for carbon taxes: creating economic incentives to protect the atmosphere. Washington, DC, World Resources Institute, ago. 1992.

FAO. Forest resources assessment 1990: tropical countries. Rome, FAO Forestry paper 112 Food and Agriculture Organization of the United Nations, 1993.

FUNATURA/ITTO. Diagnóstico e avaliação do setor florestal brasileiro. Brasília, DF, Relatorios Estaduais, 1994.

GUIMARÃES, R.J. Modernidad, medio ambiente y ética: los dilemas del mundo en desarrollo. CONFERÊNCIA INTERNACIONAL AMAZÔNIA 21: Una Agenda para un mundo sustentable Brasilia, dic. 1997. 
GOLDEMBERG, J. A simple plan to stop global warming. Princeton University Technology Review, p. 24-31, Nov./Dec. 1990.

GOULDING, M.; SMITH, N.J.H. \& MAHAR, D.J. Floods of fortune: ecology and economy along the Amazon. New York, Columbia University Press, 1995. 193p.

GTA/AMIGOS DA TERRA 1994 Políticas públicas coerentes para uma Amazônia sustentável: o desafio da inovação e o Programa Piloto. Brasília, DF, Grupo de Trabalho Amazônico e Amigos da terra (Programa Amazônia), 1994. 189p.

IDB/UNDP/TCA. Amazonia without myths. Washington, DC, Commission on Development and Environment for Amazonia, Inter-American Development Bank/United Nations Development Fund/Amazon Cooperation Treaty, 1992. 99p.

JANZEN, D.H. Tropical dry forests: the most endangered major tropical ecosystem. In: E.O. Wilson \& F.M. Peter (eds.). Biodiversity. Washington, DC, Biodiversity National Academy of Sciences, 1988, p. 130-137.

MEGGERS, B.J. Man and culture in a counterfeit paradise. Chicago, Aldine, 1971.

MITTERMEIER, R.A. \& BOWLES, I.A. The global environment facility and biodiversity conservation: lessons to date and suggestions for future action. Biodiversity and Conservation, n. 2, p. 637-655, 1993.

NAIM, M. Las intituciones: el eslabón perdido en las reformas económicas de América Latina. SEMINARIO LATINOAMERICANO SOBRE CRECIMIENTO ECONÓMICO. Bogotá, Gobierno de Colombia/Banco Mundial, 27-28 jun. 1994. $45 \mathrm{p}$.

ROJAS, M. \& CASTAÑO, C. Áreas protegidas de la cuenca del Amazonas. Bogotá, Tratado de Cooperación Amazónica/INDERENA, 1991. 213p.

RYLANDS, A.B. The status of conservation areas in the Brazilian Amazon. Washington, DC, World Wildlife Fund, 1991. 146p.

SALATI, E. The climatology and hydrology of Amazonia. In: G.T. Prance \& T. Lovejoy (eds.) Amazonia. Oxford, Inglaterra, Pergamon Press, 1985, p. 1848.

SCHNEIDER, R. The potential for trade with the Amazon in greenhouse gas reduction. LATEN Dissemination Series, The World Bank, Apr. 1993.

STONE, P.H. Forecast cloudy: the limits of global warming models. Technology Review, p. 32-40, Feb./Mar. 1992.

TCA REUNIÓN ORDINARIA DE LA COMISIÓN ESPECIAL DE TRANS- 
PORTES DE LA AMAZONÍA, 1. Anales. Quito, Secretaría Pro-Témpore del Tratado de Cooperación Amazónica, 1991.

ROOSEVELT, A.C. Chiefdoms in the Amazon and Orinoco In Chiefdoms in the Americas. In: R.D. Drennan \& C.A. Uribe (eds.). Lanham, University Press of America, 1987, p. 153-184.

TRAUMANN, T. Começa o ataque dos asiáticos (Madereiras da Ásia chegam ao Amazonas depois de deixar um rastro de destruição em outros países) Veja, p. 58-61, jun. 1997.

WRI World resources 1987. Washington, DC, World Resources Institute, 1987.

RESUMO - A DESTRUIÇÃO DOS recursos naturais da Amazônia continua aumentando, especialmente o desmatamento, apesar de a retórica dos últimos 50 anos ter mudado de conquista e exploração para desenvolvimento sustentável. Entretanto, mudanças positivas são percebidas, ainda que pequenas: o crescimento da participação popular nas decisões; o Tratado de Cooperação Amazônica, que começa a dar resultados práticos; o interesse internacional melhor orientado; existem experiências bemsucedidas e melhor documentadas sobre desenvolvimento sustentável; e muitos dos antigos mitos sobre a realidade amazônica estão sendo abandonados. O crescimento da população urbana é uma das principais mudanças percebidas. Hoje, mais de $60 \%$ de sua população é urbana e tem grande influência nas decisões políticas, ainda que não necessariamente favorável para o desenvolvimento sustentável. Neste trabalho explora-se algumas das razões pelas quais o desenvolvimento amazônico não é sustentável: entre outras, um paradigma de desenvolvimento sustentável mal definido, a persistente deficiência na identificação dos atores amazônicos e na solução de seus conflitos no planejamento regional, a fragilidade crescente das instituições públicas, a aplicação do simples crescimento econômico como estratégia dominante de desenvolvimento, o limitado acesso à educação, a desordem social. Duas ações estratégicas, que não constituem novidade, são consideradas essenciais para mudar gradativamente o padrão do desenvolvimento na região: a intensificação do uso da terra e a elevação da produtividade nas áreas já desmatadas (acima de 100 milhões de hectares na Amazônia produzem pouco ou nada); a avaliação e o pagamento em escala nacional e internacional dos serviços ambientais fornecidos pela floresta. Esses dois requisitos deverão ser acompanhados pela adoção outras estratégias bem conhecidas, como o manejo sustentável da floresta natural, o reflorestamento de áreas desmatadas não-aproveitáveis na agricultura, estabelecimento de áreas protegidas efetivamente manejadas, entre várias mais. 
ABSTRACT - AMAZON NATURAL resources destruction trends continues to increase, especially deforestation, despite changes in rethoric that during the last 50 years moved from conquest and exploitation to sustainable development. However, a few positive changes are noticeable: Amazon people's participation in decision making increased, the Amazon Treaty is starting to produce practical results, the international concerns are better oriented, there are more and better documented success stories to replicate and, several pervasive and prejudicial myths about the Amazon are being lost. Urban population growth is one of the principal changes in the Amazon. Over 60\% of the Amazon population are urban and its influence in policy making is very high and not necessarily favorable to sustainable development. In this paper are explored some of the reasons for which development is not sustainable in the Amazon: a sustainable development paradigm ill defined and poorly understood, a persistent lack of identification of actors and their conflicts in most planning exercises, growing fragility of states to organize and provide the rules of the game for development and, of course, the application of the economic growth as dominant strategy combined with lack of education and social order, corruption and other associated evils, are briefly discussed. Two strategic actions, that are not new, are considered central to gradually change the pattern of development in the Amazon: intensification of the use of the land and productivity elevation in already deforested areas (well over 100 million hectares that currently produce very little or nothing); the valuation and payment, at national and international scale, of the environmental services of the forests. These two central requirements are coupled by several other well-known and complementary possibilities such as natural forest management; establishment and management of protected areas, redefinition of success indicators for the Amazon, ecotourism etc. Education, information and participation are essential to allow democracy to play its role in sustainable development.

Marc J. Dourojeanni é especialista ambiental do Banco Interamericano de Desenvolvimento, baseado em Brasília, DF.

Trabajo presentado a la Conferencia Internacional Amazonia 21: Una Agenda para un Mundo Sustentable, Brasilia, Diciembre de 1997. Las opiniones vertidas en este paper, así cómo cualquier error u omisión, son de exclusiva responsabilidad del autor y, bajo ningún pretexto, representan la posicion oficial del Banco Interamericano de Desarrollo. 